\title{
Nonlinear optical microscopy with vibrational contrast
}

\author{
M.J. WINTERHALDER \& A. ZUMBUSCH \\ Department of Chemistry, University of Konstanz, D-78457, Konstanz, Germany
}

Key words. CARS, nonlinear optical microscopy, SRS, SFG, vibrational

spectroscopy.

\begin{abstract}
Summary
Nonlinear optical techniques which provide vibrational contrast have gained increasing attention in microscopy during the last two decades. After outlining the potential of these techniques, we give a brief introduction to coherent anti-Stokes Raman scattering, stimulated Raman scattering and sum frequency generation and discuss their suitability for contrast generation in optical microscopy. The rapid developments in these fields during the last decade have resulted in many different applications. Three exemplary application areas will therefore be presented in the last part of this manuscript.
\end{abstract}

\section{Introduction}

The generation of contrast in most optical microscopy techniques relies on the probing of electronic properties of the samples. These approaches can either employ off-resonance conditions or resonant excitation of electronic transitions (Davidson \& Abramowitz, 2002). The first type of techniques mostly probes differences in refractive indices. Examples are differential interference contrast or phase contrast microscopy. By contrast to this, the second class of methods uses spectroscopic approaches, i.e. selective light absorption or the detection of specific emission bands. In these experiments, a distinction between different sample regions is determined by their specific spectroscopic properties. Light absorption by the excitation of a molecule from its electronic ground to an electronically excited state certainly is the most widespread spectroscopic technique for contrast generation. A typical example for this approach is sample staining with hematoxylin and eosin. After staining with these dyes, sample regions which accumulate the one or the other compound then appear blue or red due to the different electronic absorption spectra of either of the labels. A very similar approach is used in modern fluorescence microscopy (see, for example, Lichtman \& Conchello, 2005). In this case, the electronic absorption is used to excite fluorescence emission. Since the fluorescence emission spectra can

Correspondence to: Andreas Zumbusch, Department of Chemistry, University of Konstanz, D-78457 Konstanz, Germany. Tel: +49-(0)7531-88-2357; fax: +49(0)7531-88-3139; e-mail: andreas.zumbusch@uni-konstanz.de themself be used for spectral distinction of compounds, fluorescence microscopy usually combines spectral selection in both absorption and emission by use of appropriate excitation wavelengths and emission filters.

From this point of view, electronic absorption spectroscopy lies at the heart of many optical microscopy techniques. Yet, the success of these techniques is owed more to the selectivity of the labelling strategies than to the spectral resolution. This is due to the fact that electronic spectral bands of most dyes are comparatively broad and featureless such that their absorptions often cover one fifth of the whole visible spectral region. Distinction of several chromophores which are simultaneously present in a microscopic experiment therefore requires special techniques.

The situation is different in the realm of vibrational spectroscopy. In contrast to electronic bands, vibrational bands are fairly narrow with respect to the useful range of vibrational frequencies, typically spanning $1 \%$ of the latter. In addition, a normal vibrational spectrum contains many more spectral features than an electronic spectrum, since even small molecules possess many vibrational eigenmodes which can be excited. Thus, vibrational spectra have long been used to identify chemical compounds on the basis of their bands in the 'fingerprint region' (Lyon et al., 1998). It therefore appears attractive to employ vibrational spectroscopy in order to generate contrast with molecular selectivity in unlabelled samples. It should, however, be noted that the richness of vibrational spectra also leads to problems in samples which contain a mixture of many unknown molecular species, which is the case for most samples of interest. Under these circumstances, the spectra of the different compounds overlap to form a complicated spectrum which is hard to interpret. Nevertheless, vibrational microscopy has been used for decades, mostly using direct infrared (IR) absorption or Raman scattering as a spectroscopic basis (Matthäus et al., 2008; Opilik et al., 2013). The choice of method depends strongly on the sample of interest, but both methods have drawbacks which motivated the search for alternative microscopy approaches generating vibrational contrast.

The aim of this short review is to briefly introduce nonlinear optical techniques providing vibrational contrast in 


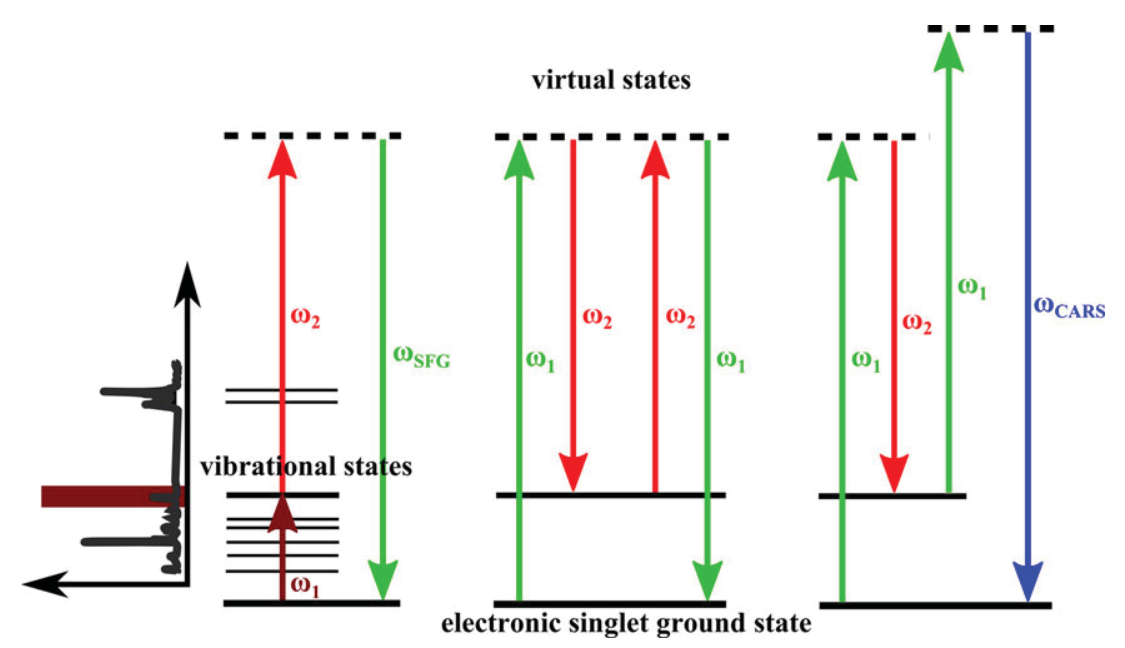

Fig. 1. Schematics showing the vibrationally resonant processes of SFG (left), SRS (middle) and CARS (right).

microscopy and to give an impression of possible applications. For more detailed information on the nonlinear optical processes coherent anti-Stokes Raman scattering (CARS), stimulated Raman scattering (SRS) and sum frequency generation (SFG) and their applications in microscopy, the reader is referred to reviews in the literature (Cheng et al., 2002; Evans \& Xie, 2008; Chung et al., 2013; Zumbusch et al., 2013).

\section{CARS, SRS and SFG microscopy}

In contrast to the linear spectroscopic techniques described above, SFG and CARS do not excite the molecule into another state. Instead, they are based on parametric processes in which the sample molecules just serve as a medium to mix excitation frequencies (see Fig. 1). Such processes can be described by an induced nonlinear polarization $P^{(n)}$. Based on their degree of nonlinearity with respect to the dependence of the signal strength on the excitation intensity, the different phenomena can be categorized either as $\chi^{(2)}$ or as $\chi^{(3)}$ processes. Here, the $\chi$ denote the nonlinear optical susceptibility as proportionality constant. A spectroscopic response, and thus also contrast generation in the microscopic application, is monitored by observing resonant enhancements of the frequency mixing process.

The simplest process discussed here is SFG. It is a $\chi^{(2)}$ process in which two excitation lasers with frequencies $\omega_{1}$ and $\omega_{2}$ are used to generate a third frequency $\omega_{\mathrm{SFG}}$. The signal intensity $I_{\mathrm{SFG}} \propto \chi^{(2)} I_{1} I_{2}$, where $I_{1}$ and $I_{2}$ denote the intensities of the exciting fields. In general, the nonlinear SFG signal can be generated in any polarizable medium, but coupling of electrons in the molecular bonding orbitals to the vibrational eigenmodes of a sample molecule will lead to a vibrational resonance enhancement of the SFG. This is observed when one of the excitation lasers excites a molecular vibrational resonance. In contrast to SFG, CARS is a $\chi^{(3)}$ process for which the signal intensity is $I_{\mathrm{CARS}} \propto \chi^{(3)} I_{1} I_{2} I_{3}$. Also, here, a resonance enhancement of a frequency mixing process in which exciting fields at $\omega_{1}, \omega_{2}$ and $\omega_{3}$ with the corresponding intensities $I_{1}, I_{2}$ and $I_{3}$ generate a signal at $\omega_{\text {CARS }}$ is used for contrast generation. The resonance condition for CARS is such that the frequency difference $\omega_{1-} \omega_{2}$ coincides with a vibrational resonance $\omega_{\text {vib }}$. Often, CARS experiments are performed in a frequency degenerate manner where $\omega_{1}=\omega_{3}$ such that only two excitation lasers are needed. SRS can be described as an $\chi^{(3)}$ process, too. Two excitation lasers are used and their frequencies are again tuned such that $\omega_{1}-\omega_{2}=\omega_{\text {vib. }}$ Yet, now one observes the change in the number of photons on either $\omega_{1}$ or $\omega_{2}$ as a signal. Stimulated Raman loss (SRL) or stimulated Raman gain (SRG) is observed when the resonance condition is fulfilled. Two important differences between the three are worth mentioning. Firstly, whereas both SFG and CARS generate signals at new frequencies, this is not the case for SRS. Secondly, the signals of the $\chi^{(2)}$ processes are only generated in the absence of molecular inversion symmetry and a symmetry break on a length scale of the excitation wavelength, i.e. typically at interfaces. By contrast, $\chi^{(3)}$ signal generation is not limited in this manner.

The differences in the nonlinear processes just described lead to peculiar experimental prerequisites for their exploitation as contrast mechanisms in optical microscopy. Common to all of them is the necessity to overlap at least two pulsed laser beams temporally and spatially in the focus. Due to the nonlinear intensity dependence of each of the processes, significant signal is only generated in the focal area. This means that all of the techniques have an inherent 3-D imaging capability with z-resolution comparable to that of a confocal microscope. Images are then generated by either raster-scanning the sample under a fixed focus or by raster-scanning the laser beams over the sample. In addition, schemes for widefield illumination have been developed (Heinrich et al., 2004; Toytman et al., 2007; Lei et al., 2011). The focusing of both excitation lasers does not pose any serious problems for CARS and SRS, since high numerical aperture objectives which are 


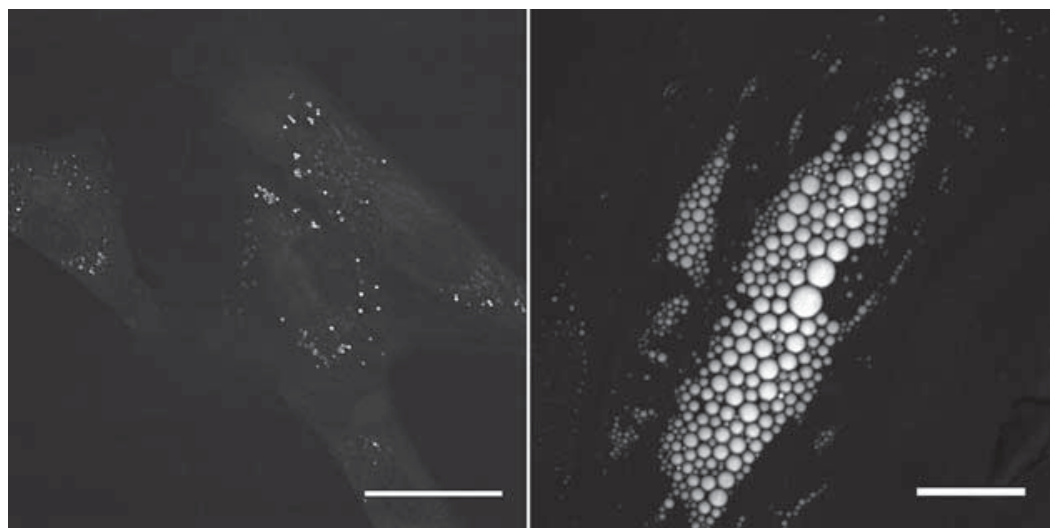

Fig. 2. CARS microscopy of LDs in mesenchymal stem cells. Left: Undifferentiated stem cells contain only a low number of small LDs. Right: A drastic change in LD number and size is observed during adipogenic differentiation of the cells. Scale bars: $30 \mu \mathrm{m}$. Images courtesy of C. Jüngst, University of Konstanz. Right image adapted from Paar et al. (2012).

colour corrected up to $1300 \mathrm{~nm}$ are commercially available. The situation is different for SFG where a visible and an IR beam have to be focused onto the same spot. In this case, one has to revert to using reflective objectives, e.g. of Cassegrain type. With respect to the detection scheme, SFG and CARS microscopy have similar requirements, since in both cases, the signal appears at a new frequency. This means that a separation of excitation and signal light by means of an optical filter is sufficient. One should note, however, that low-frequency vibrational resonances are hard to detect with these techniques. For SRS, a completely different detection scheme is necessary as one has to detect the SRL or the SRG signal at one of the excitation wavelengths. In practice, this is done by modulating one of the excitation lasers and using a lockin detection scheme. Concerning the achievable sensitivity, all nonlinear techniques discussed here are much less sensitive than fluorescence microscopy. The cross section of linear Raman scattering is orders of magnitude smaller than the electronic absorbtion cross section of a dye molecule. Due to the coherent nature of the signal generation process in SFG, SRS and CARS, the strength of the detected signal is enhanced. Still, these processes do not reach the efficiency of direct electronic excitation. In addition, the sensitivity of these nonlinear techniques is further limited by the existence of a nonresonant background. Whereas for SFG as well as for CARS, a purely electronic background exists, SRS microscopy signals may be compromised by cross-phase modulation. For strong bands such as transitions in the $\mathrm{CH}$ stretching vibration region, this does not lead to problems. The proper detection of weaker bands, however, requires more sophisticated techniques for background suppression, which are experimentally challenging.

The main advantage of the nonlinear techniques over IR absorption and spontaneous Raman scattering is the much faster image acquisition. This is possible, since in most nonlinear experiments, only a very limited range of vibrational resonances is addressed at one point in time. By contrast, IR as wells as Raman microscopy are usually performed in a multiplexing manner where either a broadband excitation is used for IR microscopy or inelastic scattering into all the vibrational modes is observed in Raman microscopy

\section{Applications of nonlinear vibrational microscopy}

Whereas CARS microscopy has seen a surge in activity over the last 15 years, SFG and SRS microscopy have both been introduced only very recently (Zumbusch et al., 1999; Ploetz et al., 2007; Freudiger et al., 2008; Nandakumar et al., 2009; Raghunathan et al., 2011). Since this also means that many more results of CARS microscopy experiments have been published, the application examples which will be discussed in the following are all from CARS microscopy. Depending on which specific question should be answered, SFG and SRS microscopy will, however, without doubt be applied in a similar manner in the future.

Certainly, the most important field for the application of CARS microscopy is the investigation of biological cells and tissues. As it has been discussed above, a number of vibrational bands can, in principle, be used to visualize the local concentration of a certain molecular species. Yet, in nearly all experiments reported to date, nonlinear vibrational microscopy has been used to image the distribution of $\mathrm{CH}-$ rich materials, mainly lipids. There are three reasons which cause these compounds to yield the strongest contrast: i) The $\chi^{(3)}$ of the respective molecules is exceptionally high; ii) the local concentration of these molecules can be very high and iii) the corresponding $\mathrm{CH}$ resonance frequencies are well separated from other resonances. Although it might appear to be a significant limitation of these methods, it turns out that many experiments of broad interest can be performed using $\mathrm{CH}$ vibrations. This is due to the fact that the subtle differences in resonance frequencies of $\mathrm{CH}$ stretches can be related to 

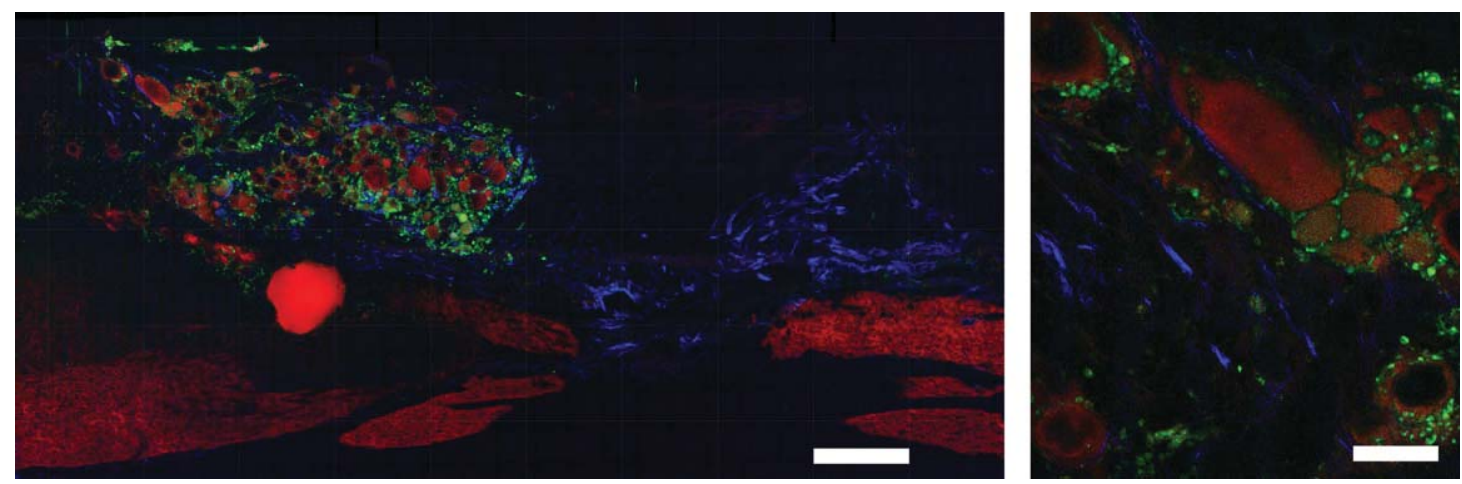

Fig. 3. Multimodal microscopy of a lesion in a spinal cord of a rat. Rats were perfusion-fixed 21 days after injury. Left: Top view on a $20 \mu \mathrm{m}$ cryosection. The CARS signal (red) shows the distribution of lipid, the TPEF signal (green) is observed from phagocytic microglia and the SHG signal (blue) is generated by collagen fibres. The image was created by stitching of multiple z-stacks. Scale bar: $5 \mathrm{~mm}$. Right: Close-up view of one z-slice. Scale bar: $1 \mathrm{~mm}$. Samples courtesy of Prof. Dr. M. Kirsch, Department of Neurosurgery, Dresden University of Technology.

antisymmetric or symmetric $\mathrm{CH}$ vibrations in $\mathrm{CH}_{2}$ - or $\mathrm{CH}_{3}$ containing compounds or to the saturated or unsaturated character of the neighbouring CC bond. Thus, it is possible to distinguish between lipids and proteins and between saturated or unsaturated compounds based on their vibrational signature in the $\mathrm{CH}$ stretching region (Chung et al., 2013).

In many cases, however, only the fact that lipids can clearly be identified based on their strong response is exploited. This and the fact that no photobleaching occurs, allows longterm observation of lipids in living cells and model organisms (Jüngst et al., 2011; Zumbusch et al., 2013). One example for such an experiment is the unraveling of the growth dynamics of lipid droplets (LDs) in live adipocytes as shown in Figure 2 (Jüngst et al. 2013). LDs have long been known as the storage site for lipids in animal and plant cells. Only during the last years, it has been recognized that apart from this, they have a lot of additional cellular functions such that they are better described as highly dynamic cellular organelles. Their dynamics also concerns their size. During adipogenic differentiation of their precursor cells, LDs grow until finally one LD occupies most of the cytoplasmic volume. Despite the importance of the LD growth, until very recently hardly anything has been know about LD growth. CARS microscopy turns out to be an ideal tool for the investigation of $\mathrm{LD}$ growth dynamics since it suits itself for long-term studies. Using this approach, it has been found that LD growth is a slow process, which is governed by the successive fusion of LDs. In this process, the content of one $\mathrm{LD}$ is transferred to the other which ultimately leads to the emergence of one unilocular LD. Since CARS microscopy allows the gathering of three-dimensional image stacks, the data can be analysed to get quantitative information about the fusion events, which, in turn, can be used to develop a biophysical model for $\mathrm{LD}$ growth.

Apart from experiments on cell culture systems, CARS microscopy has mainly been employed in biomedical investigations of tissues. An important advantage for nonlinear vi- brational microscopy in this field clearly is the fact that no labelling is necessary. This opens a perspective for the clinical use of these methods, which has motivated a large number of $e x$ vivo and in vivo experiments. An important point in these investigations is the simultaneous use of several imaging modalities at the same time (Imitola et al., 2011; Yue et al., 2011). For CARS microscopy, this is straightforward since the excitation sources employed for CARS microscopy are the same as those used for two-photon excited fluorescence microscopy and second harmonic generation (SHG) microscopy. In practice, the various signals are simply separated by the choice of appropriate optical filters. Multimodal microscopy of this type might be especially useful for investigations of neurosurgical samples. An example for this is the following of scar formation after lesions of the spinal cord (Galli et al., 2012). A major problem for wound healing here is inflammations and scar formation. Multimodal label-free imaging as shown in Figure 3 is an ideal tool for studying such processes: CARS microscopy gives excellent contrast for the myelin sheets surrounding the nerves; two-photon excited autofluorescence of the samples indicates centres of inflammation; SHG imaging in which collagen fibres show strong signal gives insight into the formation of scars. The latter is an important factor preventing the reestablishment of nervous signal transduction along the spinal cord. Experiments of this type exemplify the potential of nonlinear vibrational microscopy in clinical applications. For future experiments aiming at the application on patients, it is important to note that the excitation powers typically used for CARS microscopy are below those of normal two-photon microscopy.

Despite the fact that biological and biomedical applications currently meet a lot of interest, it is worthwhile exploring possible use of CARS microscopy as a microanalytical tool in material science. The clear difference compared to most biological samples here is the fact that for analytical investigations of this type, often the compounds present in the samples 

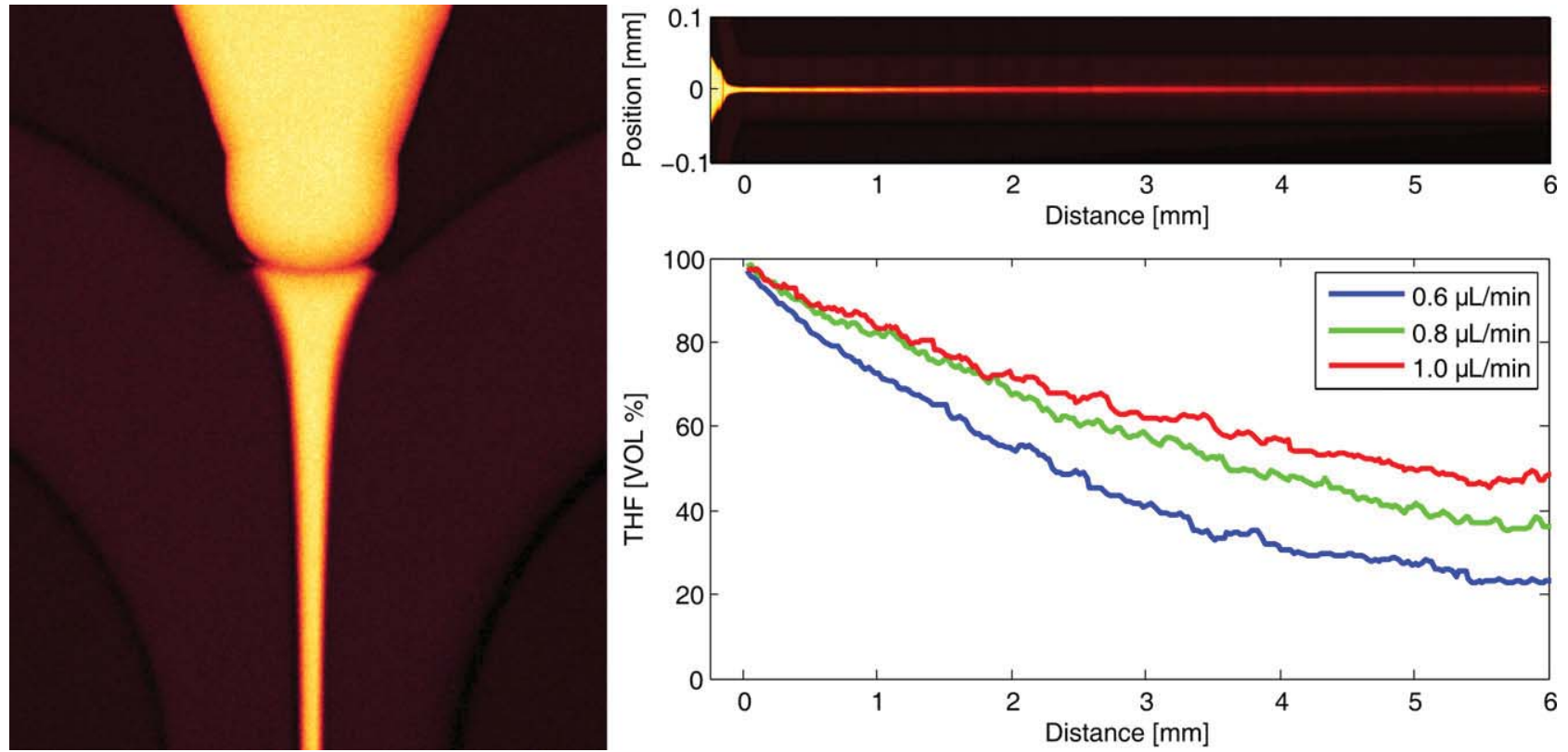

Fig. 4. CARS microscopy of microfluidic mixing of THF and water at the $\mathrm{CH}_{2}$ resonance at $2860 \mathrm{~cm}^{-1}$. Left: Close-up view of the microfluidic crossjunction. Upper right: Overview of the microfluidic device. The CARS signal of the THF decays with the progress of the mixing process. Lower right: CARS microscopy monitors the differences in THF concentration at different flow rates of THF in the mixing device. From Schütze et al. (2012).

are known and only the local concentration needs to be determined. Examples for such applications are investigations of blended materials (Chen \& Lim, 2008; Rehbinder et al., 2010). Also, processes such as reaction or mixing dynamics in microfluidic devices can be studied (Schafer et al., 2008; Schütze et al., 2012). Fluorescent labelling often is precluded here, since it will lead to an unwanted modification of the sample properties. Image acquisition in spontaneous Raman microscopy, however, is too slow to allow the fast investigation of large sample areas. This is possible with CARS microscopy which does not rely on previous sample treatment. As an example, Figure 4 shows the mixing of tetrahydrofuran (THF) with water after a microfluidic three-way junction (Schütze et al., 2012). In this case, knowledge of the local THF concentration was needed in order to understand the formation of nanoparticles which were formed from a polymeric precursor dissolved in THF.

The previous short review of the different nonlinear vibrational microscopy techniques aims at giving nonexperts an insight into this vivid field of research. The use of vibrational contrast methods was motivated. We gave a brief description of the nonlinear processes SFG, CARS and SRS and their technical implementation. On the one hand, the development of microscopy applications of these methods has largely taken advantage of spectroscopic techniques brought to perfection over many years. Their recent adaptation to optical microscopes, on the other hand, motivated many research efforts on new laser excitation sources, but most importantly prepared the ground for numerous applications. We focused on appli- cations of CARS microscopy. As examples, we showed live cell imaging of LDs for analysis of their growth dynamics, the combination of CARS microscopy with other nonlinear optical imaging modalities in neurosurgical research and finally its use as a microanalytical tool in material science.

\section{Acknowledgements}

The authors are grateful to Christian Jüngst for the provision of Figure 2 and to Prof. Dr. M. Kirsch for the sample shown in Figure 3. Funding by the German Federal Ministry of Education and Research in the frame of the MIKROQUANT project is gratefully acknowledged.

\section{References}

Chen, B.-C. \& Lim, S.-H. (2008) Optimal laser pulse shaping for interferometric multiplex coherent anti-Stokes Raman scattering microscopy. J. Phys. Chem. B 112, 3653-3661.

Cheng, J. -X., Volkmer, A.\& Xie, X.S. (2002) Theoretical and experimental characterization of coherent anti-Stokes Raman scattering microscopy. J. Opt. Soc. Am. B - Opt. Phys. 19, 1363-1375.

Chung, C.-Y., Boik, J. \& Potma, E.O. (2013) Biomolecular imaging with coherent nonlinear vibrational microscopy. Annu. Rev. Phys. Chem. 64, 77-99.

Davidson, M.W.\& Abramowitz, M. (2002) Optical Microscopy. Encyclopedia of Imaging Science and Technology. John Wiley \& Sons, Hoboken.

Evans, C.L. \& Xie, X.S. (2008) Coherent anti-Stokes Raman scattering microscopy: chemical imaging for biology and medicine. Annu. Rev. Anal. Chem. 1, 883-909. 
Freudiger, C.W., Min, W., Saar, B.G., et al. (2008) Label-free biomedical imaging with high sensitivity by stimulated Raman scattering microscopy. Science 322, 1857-1861.

Galli, R., Uckermann, O., Winterhalder, M.J., et al. (2012) Vibrational spectroscopy imaging and multiphoton microscopy of spinal cord injury. Anal. Chem. 84, 8707-8714.

Heinrich, C., Bernet, S. \& Ritsch-Marte, M. (2004) Wide-field coherent anti-Stokes Raman scattering microscopy. Appl. Phys. Lett. 84, 816818.

Imitola, J., Côté, D., Rasmussen, S., et al. (2011) Multimodal coherent anti-Stokes Raman scattering microscopy reveals microglia-associated myelin and axonal dysfunction in multiple sclerosis-like lesions in mice. J. Biomed. Opt. 16, 1-11.

Jüngst, C., Winterhalder, M. \& Zumbusch, A. (2011) Fast and long term lipid droplet tracking with CARS microscopy. J. Biophot. 4, 435-441.

Jüngst, C., Klein, M. \& Zumbusch, A. (2013) Long-term live cell microscopy studies of lipid droplet fusion dynamics in adipocytes. J. Lipid Res. 54, 3419-3429.

Lei, M., Winterhalder, M., Selm, R. \& Zumbusch, A. (2011) Video-rate wide-field CARS microscopy with collinear non-phase-matching illumination. J. Biomed. Opt. 16, 1-5.

Lichtman, J.W. \& Conchello, J.-A. (2005) Fluorescence microscopy. Nat. Methods 2, 12, 910-919.

Lyon, L.A., Keating, C.D., Fox, A.P., Baker, B.E., He, L., Nicewarner, S.H., Mulvaney, S.P. \& Natan, M.J. (1998) Raman spectroscopy. Anal. Chem. 70, 341R-361R.

Matthäus, C., Bird, B., Miljkovic, M., Chernenko, T., Romeo, M. \& Diem, M. (2008) Infrared and Raman microscopy in cell biology. Methods Cell Biol. 89, 275-308.

Nandakumar, P., Kovalev, A. \& Volkmer, A. (2009) Vibrational imging based on stimulated Raman scattering microscopy. New J. Phys. 11, 033026 .
Opilik, L., Schmid, T. \& Zenobi, R. (2013) Modern Raman imaging: vibrational spectroscopy on the micrometer and nanometer scales. Annu. Rev. Anal. Chem. 6, 379-398.

Paar, M., Jüngst, C., Steiner, N.A., et al. (2012) Remodeling of lipid droplets during lipolysis and growth in adipocytes. J. Biol. Chem. 287, $11164-$ 11173.

Ploetz, E., Laimgruber, S., Berner, S., Zinth, W. \& Gilch, P. (2007) Femtosecond stimulated Raman microscopy. Appl. Phys. B 87, 389-393.

Raghunathan, V., Han, Y., Korth, O., Ge, N.H. \& Potma, E.O. (2011) Rapid vibrational imaging with sum frequency generation microscopy. Opt. Lett. 36, 3891-3893.

Rehbinder J., Pohling C., Buckup T. \& Motzkus M. (2010) Multiplex coherent anti-Stokes Raman microspectroscopy with tailored Stokes spectrum. Opt. Lett. 35, 3721-3723.

Schafer, D., Squier, J.A., van Maarseveen, J., Bonn, D., Bonn, M. \& Müller, M. (2008) In situ quantitative measurement of concentration profiles in a microreactor with submicron resolution using multiplex CARS microscopy. J. Am. Chem. Soc. 130, 11592-11593.

Schütze, F., Stempfle, B., Jüngst, C., Wöll, D., Zumbusch, A. \& Mecking, S. (2012) Fluorescent conjugated block copolymer nanoparticles by controlled mixing. Chem. Commun. 48, 2104-2106.

Toytman, I., Cohn, K., Smith, T., Simanovskii, D. \& Palanker, D. (2007) Wide-field coherent anti-Stokes Raman scattering microscopy with non-phase-matching illumination. Opt. Lett. 32, 1941-1943.

Yue, S., Slipchenko, M.N. \& Cheng, J.-X. (2011) Multimodal nonlinear optical microscopy. Laser Photon. Rev. 5, 496-512.

Zumbusch, A., Holtom, G. R. \& Xie, X.S. (1999) Three-dimensional vibrational imaging by coherent anti-Stokes Raman scattering. Phys. Rev. Lett. 82, 4142-4145.

Zumbusch, A., Langbein, W. \& Borri, P. (2013) Nonlinear vibrational microscopy applied to lipid biology. Progr. Lipid. Res. 52, 615632. 